\title{
Histopathologic discrepancy between endoscopic forceps biopsy and final pathology and the role of transrectal ultrasound-guided core needle biopsy
}

\author{
Jin Roh ${ }^{1}$, Choong-Kyun Noh ${ }^{2}$ \\ ${ }^{1}$ Department of Pathology, ${ }^{2}$ Department of Gastroenterology, Ajou University School of Medicine, Suwon, Republic of Korea \\ Correspondence to: Choong-Kyun Noh, MD, PhD. Department of Gastroenterology, Ajou University School of Medicine, 164, World Cup-ro, \\ Yeongtong-gu, Suwon 16499, Republic of Korea. Email: cknoh23@gmail.com. \\ Provenance: This is an invited article commissioned by the Editorial Office of Annals of Translational Medicine. \\ Comment on: Liu M, Lu ZH, Wang QX, et al. Diagnostic value, safety, and histopathologic discrepancy risk factors for endoscopic forceps biopsy and \\ transrectal ultrasound-guided core needle biopsy in rectum lesions. Ann Transl Med 2019;7:607.
}

Submitted Nov 27, 2019. Accepted for publication Dec 06, 2019.

doi: $10.21037 / \mathrm{atm} .2019 .12 .52$

View this article at: http://dx.doi.org/10.21037/atm.2019.12.52

The diagnosis of gastrointestinal tract neoplasms involves histopathologic confirmation of the biopsy sample obtained from the lesion using forceps after endoscopy (1). Hence, endoscopic forceps biopsy (EFB) is an important procedure for diagnosing rectal cancer. However, the outcomes of EFB can sometimes be insufficient for a correct diagnosis for technical reasons (2). Discordant results are often observed when the biopsy result from EFB and the final pathologic diagnosis from endoscopic resection or surgical resection are inconsistent; this is called 'discrepancy'. Discrepancy can be divided into downgraded, concordant, and upgraded as follows:

(I) downgraded: when the diagnoses of the subsequent final results show a histology of less malignant potential, e.g., from high-grade dysplasia to lowgrade dysplasia, from low-grade dysplasia to nonneoplasia, or from adenocarcinoma to adenoma;

(II) concordant: when the diagnoses obtained through EFB and final results are the same;

(III) upgraded: when the diagnoses of the final specimen showed a histology of more malignant potential, e.g., from low-grade dysplasia to highgrade dysplasia, from high-grade dysplasia to adenocarcinoma, or from low-grade dysplasia to adenocarcinoma.

Regarding the stomach, a discrepancy of 33.9-49\% is reported from the study comparing the EFB outcome and the final result obtained after endoscopic resection
(3-6). On the other hand, the confirmation rate of invasive cancer from preoperative EFB of rectal lesions is reported as $58.7-67.3 \%(7,8)$, which is slightly higher than the result obtained from the study on the stomach. Among the three types of discrepancy, upgraded discrepancy is the most problematic. Patients who need endoscopic resection or surgery for cancer may receive treatment for benign disease based on the false diagnosis, which may lead to additional treatment in the future. Therefore, strategies to find risk factors and to supplement EFB are required to reduce upgrade discrepancy.

The risk factors commonly associated with upgrade discrepancy include lesion size, depressed morphology, ulceration, and whitish discoloration $(6,9,10)$. These risk factors were confirmed from stomach lesions; in comparison, there are insufficient studies on the association with discrepancy in colorectal lesions. However, it is reported that the findings of large tumor size $(>1 \mathrm{~cm})$, depressed, ulceration, or tumor necrosis in high-grade intraepithelial neoplasm can distinguish them from cancer lesions (11). These risk factors are crucial as they can predict discrepancy. For instance, when high-grade adenoma is found from EFB but the aforementioned risk factors are observed, re-biopsy or other diagnostic methods can be considered due to the probability of upgrade discrepancy. Alternatively, therapeutic methods for cancer can be considered. Regarding cancer lesions in the colorectum, surgery is the first treatment choice, not endoscopic 
resection. Therefore, reaching an accurate diagnosis for determining therapeutic measures (endoscopic resection or surgery) is more important for colorectal lesions than for gastric neoplasms.

Liu et al., in their recently published article "Diagnostic value, safety, and histopathologic discrepancy risk factors for EFB and transrectal ultrasound-guided core needle biopsy in rectum lesions", have evaluated the risk factors of discrepancy between EFB and transrectal ultrasound-guided core needle biopsy (TRUS-guided CNB) in patients with rectal neoplasm and reported that TRUS-guided CNB may serve as a safe and effective supplementary method (12).

In this study, retrospective analysis was performed on 102 patients who underwent EFB and TRUS-guided $\mathrm{CNB}$ on rectal lesions before surgery. The histopathologic discrepancy rate was $51.0 \%(52 / 102)$ in EFB and $8.8 \%$ (9/102) in TRUS-guided CNB, with the latter yielding a better outcome. In TRUS-guided CNB, the kappa value for consistency with postoperative pathology results was 0.876, which is considered good according to Cohen's kappa value. In contrast, the kappa value of FEB was 0.420 , showing relatively low consistency. Multivariate analysis with multiple logistic regression was performed to find the risk factors for discrepancy in this study, and the results presented thickness [odds ratio (OR) 1.080; 95\% confidence interval (CI), 1.021-1.142, $\mathrm{P}=0.007]$ and flat/depressed morphology of lesion (OR 0.206; 95\% CI, 0.076-0.564, $\mathrm{P}=0.002$ ) as associated factors. Furthermore, the cut-off value for thickness was determined as $13.5 \mathrm{~mm}$ using a receiver operating characteristic curve, and the sensitivity and specificity were $83.7 \%$ and $52.0 \%$, respectively. Therefore, these results suggest that TRUS-guided CNB can be useful when the thickness of the lesion is greater than $13.5 \mathrm{~mm}$.

Morphology of the lesion is reported as a risk factor for discrepancy in various studies. Depressed lesions were reported as an independent risk factor for upgrade discrepancy in several studies $(6,13,14)$. Therefore, endoscopists need to pay careful attention in cases with large lesions, surface ulceration, and depressed morphology. However, in this study, the OR (0.206) lower than that of lesions with flat/depressed morphology was observed, as the OR of elevated lesions was 1.000 . This is an associated factor analysis of the overall discrepancy (risk factors associated with histopathologic discordant diagnosis), not just the upgrade discrepancy. This is likely if it is considered that a depressed lesion was observed in one patient (11.1\%) from the discordant group of TURS-guided CNB among the patients enrolled for this study. Flat/depressed lesions are widely known as a critical warning sign suggesting colorectal invasion $(15,16)$. In this study, flat/depressed lesions accounted for $43.2 \%$ (23/52) in the discordant group of EFB. Hence, TRUS-guided CNB can be a diagnostic option for flat/depressed lesions. However, lesion thickness will be greater in elevated lesions in general. The thickness of flat/depressed lesions is predicted to be lower, and whether this might affect the accuracy of TRUS-guided CNB needs to be carefully considered.

The simplest cause of the discrepancy between EFB and final pathology is sampling error. This is because cancer cells can exist only partially in the focal lesion (17). Another cause is the inflammation that develops after EFB, which affects the cell structure and ultimately interfered with an accurate diagnosis of high-grade dysplasia or cancer (17). However, this can be different in colorectal cancer, which involves relatively large lesions and in which surgical resection plays a more important role than endoscopic resection. According to the results of the current study, the poor consistency with postoperative pathology for EFB was mainly caused by underdiagnosis of preoperative EFB. The most important histologic feature for diagnosis of colorectal cancer is detecting stromal invasion. More specifically, invasion of the muscularis mucosa should be evaluated to differentiate carcinoma in situ (intramucosal carcinoma) and invasive carcinoma. Therefore, most of the underdiagnosis for colorectal cancer is caused by a superficially sampled or poorly oriented biopsy (18). However, obtaining properly oriented tissue specimens with EFB requires considerable additional effort from clinicians. Because the sampled tissue from CNB is needle-shaped and obtained in the vertical direction, not only is the depth problem eliminated, but the issue of tissue orientation occurs during the tissue embedding also disappears. Interestingly, this study also discussed other malignant or benign lesions besides common adenocarcinoma and its precursor lesions. Because most of the subepithelial neoplastic or non-neoplastic rectal lesions are centered at the submucosa, EFB has serious limitations regarding the field of view for pathologic diagnosis (19). The authors have represented this limitation of EFB. When using the EFB, more discrepancies were identified in other types of malignant or benign lesions when comparing with the postoperative pathology type. Based on these results, it can be considered that CNB is advantageous for diagnosing deep infiltrating or deeply seated lesions. However, the inability to observe changes in the adjacent mucosa and the narrow field of CNB can limit 
the pathologists' interpretation.

Endoscopists have been using various methods to enable accurate diagnosis of lesions. There are numerous assistive methods that can supplement the endoscopist's naked eye, such as chromoendoscopy, imaging enhanced endoscopy, and confocal endoscopy. However, the final diagnostic method is histopathologic confirmation of the lesion. To achieve this, accurate histologic information should be provided to pathologists. TRUS-guided CNB can be a good alternative diagnostic tool due to these reasons, and this study presents an indication of this method (thickness and gross morphology) despite its limitations, which can be further established through additional studies.

As the authors have mentioned, this study has a few limitations and the clinical application of results should take them into account. First, this is a retrospective study in which EFB and TRUS-guided CNB were conducted on the same patients with 4-6 days of interval. Although the interval was incorporated in order to minimize the effects of these two methods, it can be still difficult to completely exclude the effects of inflammation that can follow EFB. Thus, a prospective study comparing these two methods in a randomized controlled trial is needed. It will be especially interesting to examine the results exclusively from the lesions with thickness larger than the cut-off value set by this study. Second, in addition to adenoma, high-grade intraepithelial neoplasm, and cancer, this study also included other-type benign lesion $(17,16.7 \%)$ and other-type malignant lesion $(18,17.6 \%)$. Among these, determining an accurate diagnosis with EFB might be difficult in subepithelial lesions or signet ring cell carcinoma. This is because targeting the exact location of the lesion might be challenging $(20,21)$. The role of TRUS-guided CNB in subepithelial lesions is critical and its safety is also widely known $(20,21)$. Therefore, the results from premalignant lesions and cancer lesions excluding subepithelial lesions are likely to be important. Third, the thickness is larger in the discordant group. In other words, it can be confusing that the thickness of elevated lesions would be generally greater, due to which the discordance would be higher in elevated lesions in case of EFB. However, 59.7\% (43/72) of the elevated lesions were found in the concordant group. Still, flat/depressed adenoma found in the colon is considered as an adenoma with a higher malignant potential than elevated adenoma. Flat tumor with high-grade dysplasia or invasive carcinoma especially is reported to have a smaller size than elevated adenoma $(15,22)$. Therefore, risk factor analysis on the discrepancy in flat/depressed lesions should also be followed, and whether the cut-off value for thickness can be differentially applied according to gross morphology should be examined. To achieve this, a larger number of patients should be enrolled in future studies.

In summary, the discrepancy between EFB and final histopathologic results makes the decision on treatment modality difficult for endoscopists and surgeons. In addition, since it is hard to examine deep areas of the lesions with $\mathrm{EFB}$, the discrepancy can be estimated based on various clinical clues before biopsy sampling. This study presents the potential of TRUS-guided CNB in reducing such discrepancy under certain conditions. This result can be useful for therapeutic endoscopists who need to decide between endoscopic resection and surgical resection for colorectal lesions. Although the study regarding the appropriate number of cores and the target site to adequately represent the entire lesion is critically needed for practical application, the TRUS-guided CNB reported in this study could potentially be a good alternative for reducing discrepancy between EFB and histopathologic diagnosis after treatment.

\section{Acknowledgments}

None.

\section{Footnote}

Conflicts of Interest: The authors have no conflicts of interest to declare.

Ethical Statement: The authors are accountable for all aspects of the work in ensuring that questions related to the accuracy or integrity of any part of the work are appropriately investigated and resolved.

\section{References}

1. Glynne-Jones R, Wyrwicz L, Tiret E, et al. Rectal cancer: ESMO Clinical Practice Guidelines for diagnosis, treatment and follow-up. Ann Oncol 2018;29:iv263.

2. MacDonald AW, Tayyab M, Arsalani-Zadeh R, et al. Intramucosal carcinoma on biopsy reliably predicts invasive colorectal cancer. Ann Surg Oncol 2009;16:3267-70.

3. Lee CK, Chung IK, Lee SH, et al. Is endoscopic forceps biopsy enough for a definitive diagnosis of gastric epithelial neoplasia? J Gastroenterol Hepatol 2010;25:1507-13.

4. Takao M, Kakushima N, Takizawa K, et al. Discrepancies 
in histologic diagnoses of early gastric cancer between biopsy and endoscopic mucosal resection specimens. Gastric Cancer 2012;15:91-6.

5. Sung HY, Cheung DY, Cho SH, et al. Polyps in the gastrointestinal tract: discrepancy between endoscopic forceps biopsies and resected specimens. Eur J Gastroenterol Hepatol 2009;21:190-5.

6. Noh CK, Jung MW, Shin SJ, et al. Analysis of endoscopic features for histologic discrepancies between biopsy and endoscopic submucosal dissection in gastric neoplasms: 10-year results. Dig Liver Dis 2019;51:79-85.

7. Wei XB, Gao X, Wang H, et al. More advanced or aggressive colorectal cancer is associated with a higher incidence of "high-grade intraepithelial neoplasia" on biopsy-based pathological examination. Tech Coloproctol 2012;16:277-83.

8. Tominaga K, Fujinuma S, Endo T, et al. Efficacy of the revised Vienna Classification for diagnosing colorectal epithelial neoplasias. World J Gastroenterol 2009;15:2351.

9. Kim YJ, Park J, Kim JH, et al. Histologic diagnosis based on forceps biopsy is not adequate for determining endoscopic treatment of gastric adenomatous lesions. Endoscopy 2010;42:620-6.

10. Choi CW, Kim HW, Shin DH, et al. The risk factors for discrepancy after endoscopic submucosal dissection of gastric category 3 lesion (low grade dysplasia). Dig Dis Sci 2014;59:421-7.

11. Bujanda L, Cosme A, Gil I, Arenas-Mirave JI. Malignant colorectal polyps. World J Gastroenterol 2010;16:3103-11.

12. Liu M, Lu ZH, Wang QX, et al. Diagnostic value, safety, and histopathologic discrepancy risk factors for endoscopic forceps biopsy and transrectal ultrasound-guided core needle biopsy in rectum lesions. Ann Transl Med 2019;7:607.

13. Cho SJ, Choi I, Kim C, et al. Risk of high-grade dysplasia

Cite this article as: Roh J, Noh CK. Histopathologic discrepancy between endoscopic forceps biopsy and final pathology and the role of transrectal ultrasound-guided core needle biopsy. Ann Transl Med 2020;8(4):65. doi: 10.21037/ atm.2019.12.52 or carcinoma in gastric biopsy-proven low-grade dysplasia: an analysis using the Vienna classification. Endoscopy 2011;43:465-71.

14. Ryu DG, Choi CW, Kang DH, et al. Clinical outcomes of endoscopic submucosa dissection for high-grade dysplasia from endoscopic forceps biopsy. Gastric Cancer 2017;20:671-8.

15. Kudo S. Depressed type of colorectal cancer. Endoscopy 1995;27:54-7.

16. Tsuda S, Veress B, Toth E, et al. Flat and depressed colorectal tumours in a southern Swedish population: a prospective chromoendoscopic and histopathological study. Gut 2002;51:550-5.

17. Kato M, Nishida T, Tsutsui S, et al. Endoscopic submucosal dissection as a treatment for gastric noninvasive neoplasia: a multicenter study by Osaka University ESD Study Group. J Gastroenterol 2011;46:325-31.

18. Fleming M, Ravula S, Tatishchev SF, et al. Colorectal carcinoma: Pathologic aspects. J Gastrointest Oncol 2012;3:153-73.

19. Kim TO. Colorectal subepithelial lesions. Clin Endosc 2015;48:302-7.

20. Adler DG, Muthusamy VR, Ehrlich DS, et al. A multicenter evaluation of a new EUS core biopsy needle: Experience in 200 patients. Endosc Ultrasound 2019;8:99-104.

21. Antonini F, Delconte G, Fuccio L, et al. EUS-guided tissue sampling with a 20 -gauge core biopsy needle for the characterization of gastrointestinal subepithelial lesions: a multicenter study. Endosc Ultrasound 2019;8:105-10.

22. Teixeira CR, Tanaka S, Haruma K, et al. Flat-elevated colorectal neoplasms exhibit a high malignant potential. Oncology 1996;53:89-93. 